\title{
Improvement of stability conditions, accuracy and uniqueness of penalty approach in contact modeling
}

\author{
Tomasz Bednarek • Piotr Kowalczyk
}

Received: 8 February 2012 / Accepted: 29 July 2012 / Published online: 22 August 2012

(C) The Author(s) 2012. This article is published with open access at Springerlink.com

\begin{abstract}
The main objective of this paper is to improve stability conditions, uniqueness and convergence of numerical analysis of metal forming processes with contact constraints enforced by the penalty method. A commonly known drawback of this approach is the choice of penalty factor values. When assumed too low, they result in inaccurate fulfillment of the constraints while when assumed too high, they lead to ill-conditioning of the equations system which affects stability and uniqueness of the solution. The proposed modification of the penalty algorithm consists in adaptive estimation of the penalty factor values for the particular system of finite element equations and for the assumed allowed inaccuracy in fulfillment of the contact constraints. The algorithm is tested on realistic examples of sheet metal forming. The finite element code based on flow approach formulation (for rigid-plastic and rigid-viscoplastic material model) has been used.
\end{abstract}

Keywords Contact modeling · Penalty approach . Metal forming $\cdot$ Deep drawing

T. Bednarek · P. Kowalczyk

Institute of Fundamental Technological Research, Polish Academy

of Sciences, Pawińskiego 5b, 02-106 Warsaw, Poland

P. Kowalczyk

e-mail: pkowalcz@ippt.gov.pl

T. Bednarek $(\varangle)$

Kazimierz Wielki University, Chodkiewicza 30,

85-064 Bydgoszcz, Poland

e-mail: bednarek@ippt.gov.pl

\section{Introduction}

An inherent feature of all analytical and numerical formulations of metal forming processes is the presence of contact constraints between the highly deformable workpiece and the forming tools, usually treated as rigid bodies. Two methods of including the constraints in the analysis are commonly accepted: the Lagrange multipliers method and the penalty method [1]. The first, although more accurate, is considered inconvenient as it increases the size of the equations system; besides, the size may vary from iteration to iteration in the solution algorithm. Thus the most common method employed is the latter, i.e. the penalty approach. In it, the contact constraints are included in the potential energy functional as quadratic forms with coefficients called penalty factors. Assuming the penalty factors large enough one can enforce approximate fulfillment of the contact constraints with good accuracy.

Unfortunately, when the numerical methods (e.g. FEM) are applied, too large values of penalty factors result in ill-conditioning of the system of equations which leads to unstable and non-unique solutions and bad convergence of the iterations schemes. The right choice of the penalty factor values appears thus to be a crucial issue in the correct formulation of the problem. In many commercial and academic codes the penalty factor is assumed constant during the whole forming process and selected arbitrarily, according to the engineer's experience. Typically, it is assumed three to six orders of magnitude above the magnitude of the highest diagonal term in the coefficient matrix of the system of equations. This estimation may not always be good. Let us mention here the case of sheet metal forming analysis, when the sheet stiffness in the in-plane and normal directions differ significantly, and to an even more extent the flow approach formulation of this problem [2-4], in which the diagonal 
terms of the coefficient matrix of the equations system have reasonably different magnitudes in different areas of the sheet model and in different stages of the forming process. Stability and uniqueness of flow approach algorithm in sheet metal forming simulations were studied e.g. in [5].

Issues of accuracy of penalty methods in contact analysis were investigated in [6,7]. Penalty parameter tuning techniques have also been considered in [8,9]. Basic regularization through an associated area and implementation of micromechanical contact models have been introduced in [10-12]. Some modifications of contact algorithm employing penalty approach were proposed in the recent work [13].

We propose a modification of the well known penalty approach in contact modeling. Our goal is to find a relationship which allows to calculate the best value of the penalty factor for given conditions. It should be noted that the penalty factor does not need to be the same for all nodes, nor to remain constant in all iterations or time steps. In the proposed approach, we only assume a limit value of the allowed inaccuracy of contact modeling (i.e. allowed tool penetration by workpiece nodes) instead of blindly assuming an abstract value of penalty factor. Penalty factors are estimated at each time instant and iteration for each node remaining in contact with tools on the basis of the current geometry and material parameters and the assumed acceptable penetration value. The resulting precision in the contact modeling preserves approximately the assumed limit value.

The numerical tests performed show good and promising results, see Sect. 3. Noticeable decrease in the condition number of the system of equations is observed. Besides, the method does not increase the number of equilibrium iterations.

\section{Methods}

In finite element modelling, the discretized contact constraints between the deformable workpiece nodes and rigid tool surfaces can be written as

$D_{k i} u_{i}-\hat{u}_{k}=0 \quad$ or $\quad \mathbf{D u}-\hat{\mathbf{u}}=\mathbf{0}$

where $\mathbf{u}=\left\{u_{i}\right\}(i=1, \ldots, N)$ is the nodal displacement vector, $\hat{\mathbf{u}}=\left\{\hat{u}_{k}\right\}(k=1, \ldots, M)$ is an array of prescribed displacements normal to the rigid surface (i.e. actual distances to the surface) at the nodes whose motion is constrained by contact constraints, while $\mathbf{D}=\left[D_{k i}\right]$ is a matrix of directional cosines of the normal directions at these locations (for nodes that are not involved in the $k$ th contact constraint, the corresponding terms in the $k$ th row of $\mathbf{D}$ are zero). Unless multiple contact constraints at particular nodes are active, the matrix $\mathbf{D}$ is orthogonal, i.e.

$$
D_{k i} D_{l i}=\delta_{k l} \quad \text { or } \quad \mathbf{D D}^{T}=\mathbf{I}_{M \times M} \text {. }
$$

\subsection{Penalty method in contact modelling}

Let us shortly recall the classical penalty approach to contact modelling in discrete systems. It consists in supplementing the potential energy functional by terms that represent the additional 'penalty energy' of contact constraints. The unconstrained functional in the discrete FE formulation for a nonlinear elastostatic system has the form

$\Pi(\mathbf{u})=G(\mathbf{u})-q_{i} u_{i}$,

where $\mathbf{q}$ is the nodal load vector while $G(\mathbf{u})$ is the strain energy. In inelastic systems the form is similar except that $G$ is expressed in terms of displacement increments and additional state parameter fields, but for clarity of presentation we will keep considering it in the above form. Including contact constraints (for simplicity of presentation, let us neglect friction forces), we can write [14]

$\Pi(\mathbf{u})=G(\mathbf{u})-q_{i} u_{i}+\sum_{k=1}^{M} \frac{1}{2} \epsilon_{k}\left(D_{k i} u_{i}-\hat{u}_{k}\right)\left(D_{k j} u_{j}-\hat{u}_{k}\right)$,

where $\epsilon_{k}$ denotes penalty factor related to $k$ th constraint. The condition of stationarity of the first derivative,

$\frac{\partial \Pi}{\partial u_{i}}=0$,

leads to the nonlinear system of equations

$$
\frac{\partial G}{\partial u_{i}}+\sum_{k=1}^{M} \epsilon_{k} D_{k i} D_{k j} u_{j}=q_{i}+\sum_{k=1}^{M} \epsilon_{k} D_{k i} \hat{u}_{k} .
$$

The system (6) is solved in iterations according to the Newton-Raphson scheme. Denoting by $\tilde{u}_{i}$ an already known approximate solution, its correction $\Delta u_{i}$ is sought for by solving the following linear system of equations,

$$
\left(K_{i j}+\sum_{k=1}^{M} \epsilon_{k} D_{k i} D_{k j}\right) \Delta u_{j}=\Delta q_{i}+\sum_{k=1}^{M} \epsilon_{k} D_{k i} \Delta \hat{u}_{k},
$$

where $K_{i j}, \Delta q_{i}$ and $\Delta \hat{u}_{k}$ are the tangent stiffness matrix of the unconstrained system, residual forces, and prescribed corrections of contact-constrained displacements, respectively:

$$
\begin{gathered}
K_{i j}=\left.\frac{\partial^{2} G}{\partial u_{i} \partial u_{j}}\right|_{u_{l}=\tilde{u}_{l}}, \quad \Delta q_{i}=q_{i}-\left.\frac{\partial G}{\partial u_{i}}\right|_{u_{l}=\tilde{u}_{l}}, \\
\Delta \hat{u}_{k}=\hat{u}_{k}-D_{k j} \tilde{u}_{j} .
\end{gathered}
$$

In the particular case of a linear elastic problem $G=K_{i j} u_{i} u_{j}$, $\Delta q_{i}=q_{i}-K_{i j} u_{j}$ and $K_{i j}$ is a constant matrix.

Resuming, we come up with the system of equations in which the tangent stiffness matrix is increased by locally nonzero terms proportional to the penalty factors. Accordingly, 
the right-hand side vector is shifted by such terms, too. The system can be written in a compact matrix form as

$$
\left(\mathbf{K}+\mathbf{D}^{T} \boldsymbol{\epsilon} \mathbf{D}\right) \Delta \mathbf{u}=\Delta \mathbf{q}+\mathbf{D}^{T} \boldsymbol{\epsilon} \Delta \hat{\mathbf{u}}
$$

where $\epsilon$ is an $M \times M$ diagonal matrix containing the penalty factors.

The penalty method does not extend the system of equations - the number of unknowns remains the same as in the unconstrained problem. It is clear from the form of (9) that if values of $\epsilon_{k}$ are large enough, the solution should fulfill the equilibrium equations and the contact constraints with good accuracy. However, taking their values too high leads to ill-conditioning of the coefficient matrix in the system of equations, which has known undesired consequences for the solution stability. The question how to choose the right values is very difficult to answer. Typically, the same value $\epsilon$ is assumed for all $M$ constraints, and it is taken as a number that dominates by 3-6 orders of magnitude over the highest diagonal term in $\mathbf{K}$. Frequently, this approximation appears much higher than it is necessary from the point of view of required accuracy of contact constraints. Particularly this happens if the constrained displacement d.o.f. has much lower stiffness than other d.o.f.'s in the model, which is a frequent case e.g. in beam or shell structures, or in the case elastic-plastic material models. It is thus very desirable to be able to adaptively estimate the penalty factors adjusting them to the particular FE system of equations for an assumed accuracy level.

\subsection{The proposed modification}

In this section, the modification of penalty approach method is proposed. The main idea is to estimate the penalty factors $\epsilon_{k}$, adjusting their values to current stiffness and load conditions of the model and to an assumed accuracy of contact modelling. It is assumed that the penalty factors $\epsilon_{k}$ differ at different locations (for different discrete node-to-surface contact constraints $k=1, \ldots, M)$ and at different time steps or even equilibrium iterations.

Let us start from a simple one-dimensional example. The model shown in Fig. 1 is considered; $k$ is a stiffness of the spring, $q$ is the exciting force, $\hat{u}$ is the assumed value of displacement (a restriction resulting from the contact constraint), $\epsilon$ is the penalty factor and $\delta$ is the limit depth of penetration.

Let us rewrite (7) for this simple one-dimensional case:

$(k+\epsilon) u=q+\epsilon \hat{u}$.

The displacement $u$ is thus given as

$u=\frac{q+\epsilon \hat{u}}{k+\epsilon}$

For large values of $\epsilon$, the value of displacement $u$ is close to the value of $\hat{u}$.

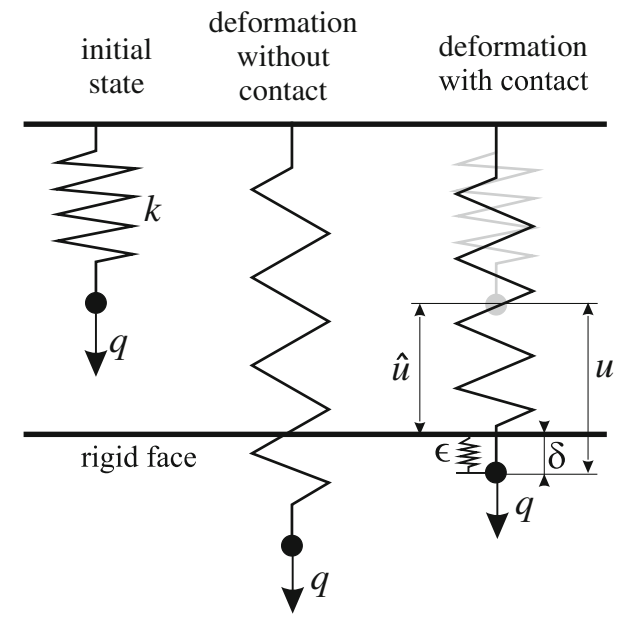

Fig. 1 Elastic spring with contact constraint

Let us now introduce the allowed inaccuracy (penetration depth) $\delta$. It is required that

$u-\hat{u} \leq \delta$

This inequality is fulfilled for only large enough values of $\epsilon$. To compute its limit value, let us assume the worst allowed result, i.e.

$u-\hat{u}=\delta$.

Substituting the relation (11) to Eq. (13) we get after transformations

$\epsilon=\frac{q-k(\hat{u}-\delta)}{\delta}$.

For this penalty factor value the allowed inaccuracy is preserved. It is clear that the higher required accuracy (i.e. the smaller value of $\delta$ ), the higher value of $\epsilon$ is required.

Let us now pass to the general case, i.e. consider the system of Eq. (9) solved at each Newton-Raphson iteration.

Let us define the allowed inaccuracy of contact modeling (penetration) as a vector $\delta=\left\{\delta_{k}\right\}, k=1, \ldots, M$. Thus, in the worst case we have

$\mathbf{D} \Delta \mathbf{u}-\Delta \hat{\mathbf{u}}=\delta$.

Substituting this to (9) one obtains

$\mathbf{K} \Delta \mathbf{u}+\mathbf{D}^{T} \boldsymbol{\epsilon} \boldsymbol{\delta}=\Delta \mathbf{q}$.

Premultiplying the above by $\mathbf{D}$ and employing the orthogonality condition (2) one comes to the following form

$\boldsymbol{\epsilon} \boldsymbol{\delta}=\mathbf{D}(\Delta \mathbf{q}-\mathbf{K} \Delta \mathbf{u})$.

Rewriting this in the index notation and recalling that $\epsilon$ is a diagonal matrix, we can finally write the expression for the penalty factors $\epsilon_{k}, k=1, \ldots, M$, as

$\epsilon_{k}=\frac{D_{k i}\left(\Delta q_{i}-K_{i j} \Delta u_{j}\right)}{\delta_{k}} \quad$ (no summation over $k$ ). 
Comparing this result to that of our 1 d.o.f. example (14), we can see an inconvenience: there are unknown terms $\Delta u_{j}$ on the right hand side of Eq. (18). These are the nodal displacement corrections that are unknowns to be found. However, let us stress at this point that we are not actually interested in exact values of $\epsilon_{k}$ that ensure precise fulfillment of equation (15). What we are actually looking for are estimated values of the penalty factors that allow the solution to approximately preserve the inaccuracy of contact modeling expressed by (15). Thus, it is indeed sufficient to substitute for $\Delta u_{j}$ in Eq. (18) any good approximate of this vector.

Our proposition to evaluate this approximate is as follows. Since in the convergent iteration scheme the subsequent corrections tend to zero, it is proposed to set $\Delta u_{j}=0$, except for the nodes where active contact constraints apply-there $\Delta u_{j}$ are set to simple orthogonal projection vectors of the current node position onto the contact surface. This can be written in a compact form as

$\Delta u_{j} \approx D_{l j} \Delta \hat{u}_{l}, \quad l=1, \ldots, M$,

i.e. the formula (18) can be rewritten in an approximate form as

$\epsilon_{k}=\frac{D_{k i}\left(\Delta q_{i}-K_{i j} D_{l j} \Delta \hat{u}_{l}\right)}{\delta_{k}}$ (no summation over $k$ ).

The approximation (19) will surely be sufficient in further iterations, when the computed corrections are indeed small. In the first iteration, the argumentation for the above choice of the approximate of $\Delta u_{j}$ may not be convincing and the classical penalty approach should be generally advised as the safe choice. Another option is to apply a special startup strategy, like the one recently proposed in [13]. However, numerical examples presented further show that satisfactory results may be obtained even in the first equilibrium iteration upon introduction of the above approximation.

Let us finally comment on the rarely met case when a node has an active contact constraint at more than one surface (i.e. it lies on an edge or a vertex of rigid surfaces). In such a case the orthogonality condition (2) does not hold and the system (17) takes the form

$$
\tilde{\mathbf{I}} \boldsymbol{\epsilon}=\mathbf{D}(\Delta \mathbf{q}-\mathbf{K} \Delta \mathbf{u}) .
$$

where $\tilde{\mathbf{I}}=\mathbf{D D}^{T}$. One has then use Eq. (18) to compute a temporary array of, say, $\epsilon_{k}^{\prime}$ and solve the following system of $M$ equations:

$\tilde{I}_{k l} \epsilon_{l}=\epsilon_{k}^{\prime}$.

This is generally not a difficult task, as the matrix $\tilde{\mathbf{I}}$ is usually nearly diagonal (has only few off-diagonal terms) and close to I. If, however, the considered edge (or vertex) is formed by two (or three) nearly coplanar segments of the tool surface (which frequently happens when smooth surfaces are discretized with finite elements), it is advised to rather apply one of the known smoothing techniques than to strictly employ the above equations.

\subsection{Penalty method for flow approach formulation in sheet metal forming}

Let us now discuss the particular formulation of FE analysis for which the modified penalty method has been implemented and tested. This is the flow approach formulation in sheet metal forming [2-4]. The approach is based on the assumption that the sheet material obeys the rigid-viscoplastic material model $[15,16]$.

In this model stresses are calculated from the constitutive equation

$\sigma_{i j}=s_{i j}+p \delta_{i j}, \quad s_{i j}=2 \mu^{*} \dot{\varepsilon}_{i j}$

where $s_{i j}$ is the Cauchy stress deviator, $p$ denotes the mean stress computed from the plane stress condition and $\dot{\varepsilon}_{i j}$ is the strain rate assumed equal to the deviatoric plastic strain rate

$\dot{\varepsilon}_{i j}=\frac{1}{2}\left(v_{i, j}+v_{j, i}\right)$.

The constitutive viscosity coefficient $\mu^{*}$ is determined from the viscoplastic flow rule [16]

$\mu^{*}=\frac{\bar{\sigma}}{3 \dot{\bar{\varepsilon}}}=\frac{1}{3 \dot{\bar{\varepsilon}}}\left[\sigma_{y}+\left(\frac{\dot{\bar{\varepsilon}}}{\gamma}\right)^{\frac{1}{n}}\right]$

Here, $\sigma_{y}$ is the current static uniaxial tensile yield stress,

$\bar{\sigma}=\sqrt{\frac{3}{2} s_{i j} s_{i j}}, \quad \dot{\bar{\varepsilon}}=\sqrt{\frac{2}{3} \dot{\varepsilon}_{i j} \dot{\varepsilon}_{i j}}$

are the equivalent stress and the effective inelastic strain rate, respectively, and $\gamma, n$ are physical parameters of the rigidviscoplastic model used. For plastic materials with strain hardening, the yield limit $\sigma_{y}=\sigma_{y}(\bar{\varepsilon})$ and the effective inelastic strain $\bar{\varepsilon}$ has to be computed as the time integral of $\dot{\bar{\varepsilon}}$. Clearly, the viscosity coefficient $\mu^{*}$ is a function of the strain rate $\dot{\varepsilon}_{i j}$.

The formal analogy between the above plastic flow equations and a formulation of incompressible plane stress elasticity allows to solve the pure plastic flow problem with a numerical code developed for nonlinear incompressible elasticity. Rates of large plastic strains are treated in the same way as elastic strains, while the stiffness matrix is replaced by viscosity matrix. Incompressibility is a source of numerical problems in a general case, however, in sheet metal forming, where the shell theory is used, it is easily included in the plane stress assumption so that constant volume of the shell section is preserved. 
Introducing the standard FE interpolation of shell displacements (see $[3,17]$ for details) and applying it to the virtual work equations together with the constitutive formulation presented above, we obtain the following nonlinear equation system for the vector of generalized (i.e. including also rotational degrees of freedom) nodal velocities $\dot{\mathbf{u}}$

$$
\mathbf{K}^{*}(\dot{\mathbf{u}}) \dot{\mathbf{u}}=\mathbf{q}
$$

in which $\mathbf{K}^{*}$ and $\mathbf{q}$ denote the viscosity matrix and the external force vector, respectively. The dependence of the matrix $\mathbf{K}^{*}$ on the solution $\dot{\mathbf{u}}$ is due to the dependence of $\mu^{*}$ on strain rate $\dot{\varepsilon}_{i j}$.

In the case of contact, the penalty approach can be easily adopted in the flow approach simulation. At a typical time step $\Delta t$, starting at time $t$, the contact constraints have the form

$D_{k i}\left(u_{i}^{t}+\dot{u}_{i} \Delta t\right)-\hat{u}_{k}=0$.

After transformations, we obtain the following nonlinear system of equations:

$$
\begin{aligned}
& \left(K_{i j}^{*}+\sum_{k=1}^{M} \epsilon_{k} D_{k i} D_{k j} \Delta t\right) \dot{u}_{j} \\
& =q_{i}+\sum_{k=1}^{M} \epsilon_{k} D_{k i}\left(\hat{u}_{k}-D_{k j} u_{j}^{t}\right) .
\end{aligned}
$$

In the Newton-Raphson iteration scheme, given the approximate solution $\tilde{\dot{u}}_{i}$, the following system for the correction $\Delta \dot{u}_{i}$ is solved

$$
\left(K_{i j}+\sum_{k=1}^{M} \epsilon_{k} D_{k i} D_{k j} \Delta t\right) \Delta \dot{u}_{j}=\Delta q_{i}+\sum_{k=1}^{M} \epsilon_{k} D_{k i} \Delta \hat{u}_{k},
$$

where the tangent viscosity matrix of the unconstrained system $K_{i j}$, the residual force vector $\Delta q_{i}$ and the prescribed corrections of contact-constrained displacements $\Delta \hat{u}_{k}$ at the considered time increment are expressed as

$$
\begin{aligned}
K_{i j} & =K_{i j}^{*}+\frac{\partial K_{i k}^{*}}{\partial \dot{u}_{j}} \dot{u}_{k}, \\
\Delta q_{i} & =q_{i}-K_{i j}^{*}(\tilde{\dot{\mathbf{u}}}) \tilde{\dot{u}}_{j}, \\
\Delta \hat{u}_{k} & =\hat{u}_{k}-D_{k j}\left(u_{j}^{t}+\tilde{\dot{u}}_{j} \Delta t\right) .
\end{aligned}
$$

We can notice the striking similarity in the form of the systems (29) and (7). This allows to easily transform the formulae of the modified penalty approach developed in Sect. 2.2 in the considered flow approach formulation. Introducing the allowed inaccuracy vector $\delta_{k}$ we can write down the worst-case inaccuracy condition as

$D_{k i} \Delta \dot{u}_{i} \Delta t-\Delta \hat{u}_{k}=\delta_{k}$.

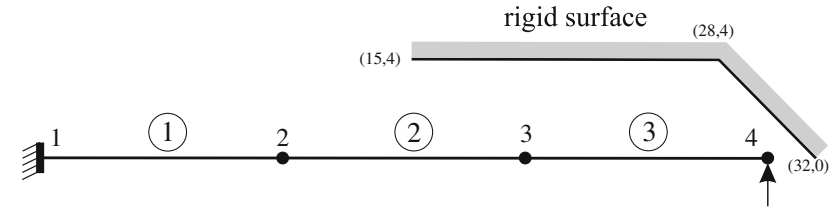

Fig. 2 Beam with contact constraints

Substituting it to (29) we obtain

$K_{i j} \Delta \dot{u}_{j}+\sum_{k=1}^{M} \epsilon_{k} \delta_{k} D_{k i}=\Delta q_{i}$

which, after transformations known from Sect. 2.2 leads to the following formulae for the penalty factors $\epsilon_{k}$ :

$\epsilon_{k}=\frac{D_{k i}\left(\Delta q_{i}-K_{i j} \Delta \dot{u}_{j}\right)}{\delta_{k}}$.

\section{Numerical examples}

\subsection{Restrained four-node beam}

To illustrate the formulation of modified penalty approach in contact modeling, let us consider the unilaterally restrained beam shown in Fig. 2. The beam is $30 \mathrm{~mm}$ long and its crosssection is a $1 \times 1 \mathrm{~mm}^{2}$. The Young modulus of the material is $E=210 \mathrm{GPa}$. The load force acting on the beam's free end is $20 \mathrm{~N}$ and its deflection is constrained by two rigid surfaces depicted in Fig. 2.

The beam is discretized with three equal two-node finite elements with two displacement and one rotation d.o.f. at each node. The stiffness matrix of a single finite element is given by

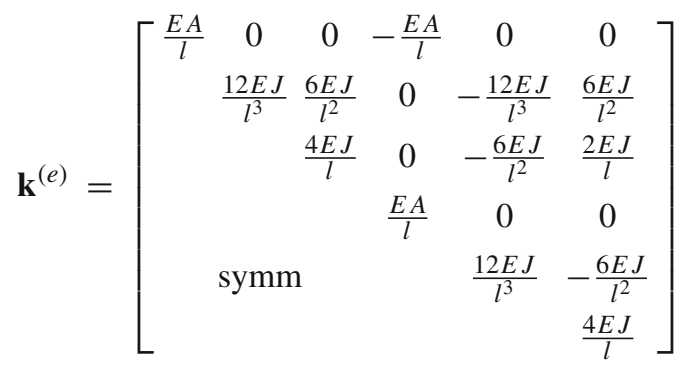

where $l$ is the element's length, $A$ and $J$ are the cross-section area and its moment of inertia, respectively.

The global stiffness is assembled from element matrices. After imposing the boundary conditions at node 1 and substituting numerical data, ${ }^{1}$ it reads

\footnotetext{
${ }^{1}$ For clarity of visualization, units are skipped in the following formulae; the units of stiffness matrix are $(\mathrm{GPa})$, displacements $(\mathrm{mm})$ and forces $(\mathrm{kN})$.
} 


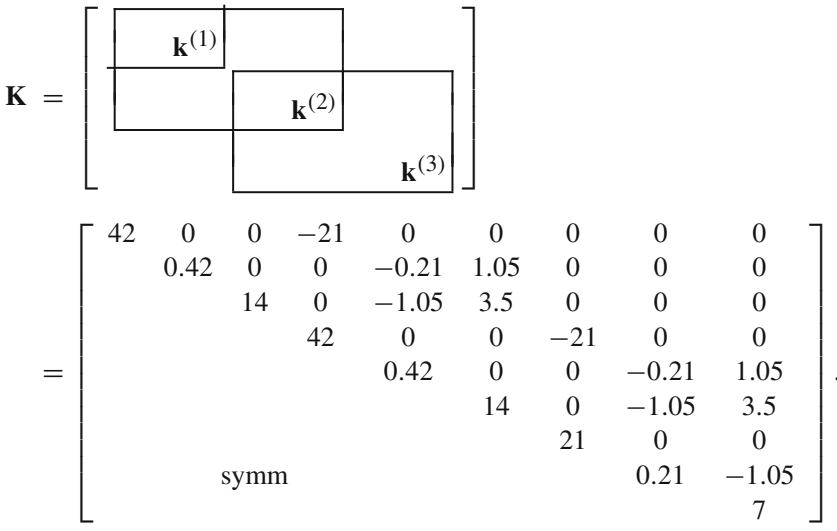

The load vector has the following components:

$\mathbf{q}=\left[\begin{array}{lllllllll}0 & 0 & 0 & 0 & 0 & 0 & 0 & 0.02 & 0\end{array}\right]^{T}$

In the first iteration no contact constraints are active and the unconstrained system of equilibrium equations $\mathbf{K u}=\mathbf{q}$ is solved. The resulting nodal displacement vector is

$$
\mathbf{u}=\left[\begin{array}{lllllllll}
0 & 1.524 & 0.286 & 0 & 5.333 & 0.457 & 0 & 10.286 & 0.514
\end{array}\right]^{T}
$$

The beam deflection for this case is shown in Fig. 3a.

In the discrete model, contact constraints affect displacements of nodes 3 and $4(M=2)$. The matrix of normal directions and the vector of allowed normal displacements in the constraint Eq. (1) have the form

$\mathbf{D}=\left[\begin{array}{llllllccc}0 & 0 & 0 & 0 & 1 & 0 & 0 & 0 & 0 \\ 0 & 0 & 0 & 0 & 0 & 0 & 0.707 & 0.707 & 0\end{array}\right]$.

Since the solution (39) violates both the constraints, in the next iteration we need to solve the penalized system (9) i.e.

$$
\left(\mathbf{K}+\mathbf{D}^{T} \boldsymbol{\epsilon} \mathbf{D}\right) \Delta \mathbf{u}=\Delta \mathbf{q}+\mathbf{D}^{T} \boldsymbol{\epsilon} \Delta \hat{\mathbf{u}}
$$

in which the approximate solution $\tilde{\mathbf{u}}$ is the previous solution (39), the matrix $\mathbf{K}$ remains the same as in the first iteration, $\Delta \mathbf{q}=\mathbf{q}-\mathbf{K} \tilde{\mathbf{u}}=\mathbf{0}$, and

$\Delta \hat{\mathbf{u}}=\hat{\mathbf{u}}-\mathbf{D} \tilde{\mathbf{u}}=\left[\begin{array}{l}-1.333 \\ -5.859\end{array}\right]$

In the classical penalty approach, the penalty factors would be assumed at the level of, say, $10^{3}$ times the highest diagonal element of $\mathbf{K}$, i.e. $\epsilon_{k}=42,000$. Let us now estimate them using the presented approach. The assumed inaccuracy of the contact modeling is taken for all constraints as $\delta=0.0001 \mathrm{~mm}$.

From (20) we obtain

$$
\boldsymbol{\epsilon}=\left[\begin{array}{ll}
\epsilon_{1} & \\
& \epsilon_{2}
\end{array}\right]=\left[\begin{array}{cc}
-3,100 & 0 \\
0 & 619,380
\end{array}\right] \text {. }
$$

Step 1

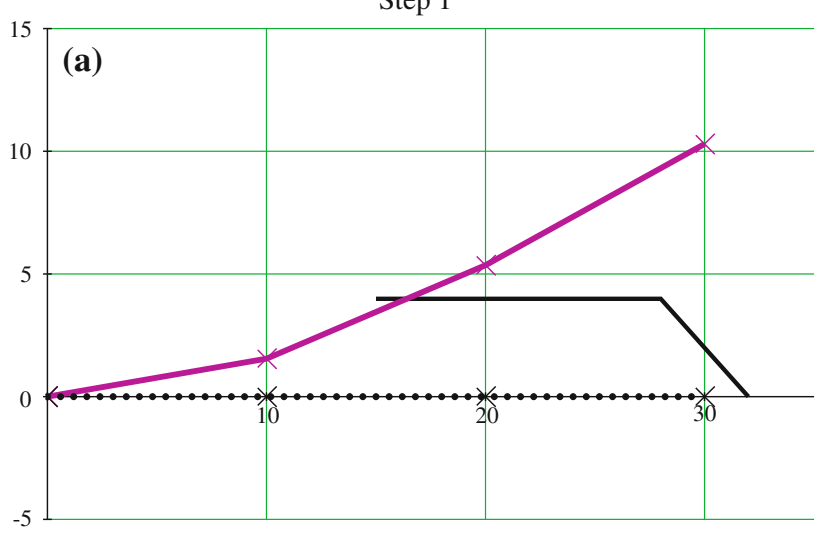

Step 2

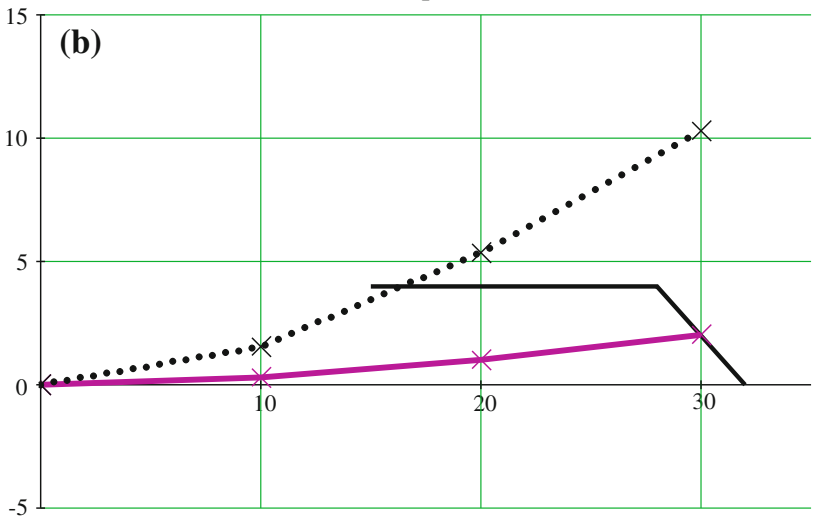

Step 3

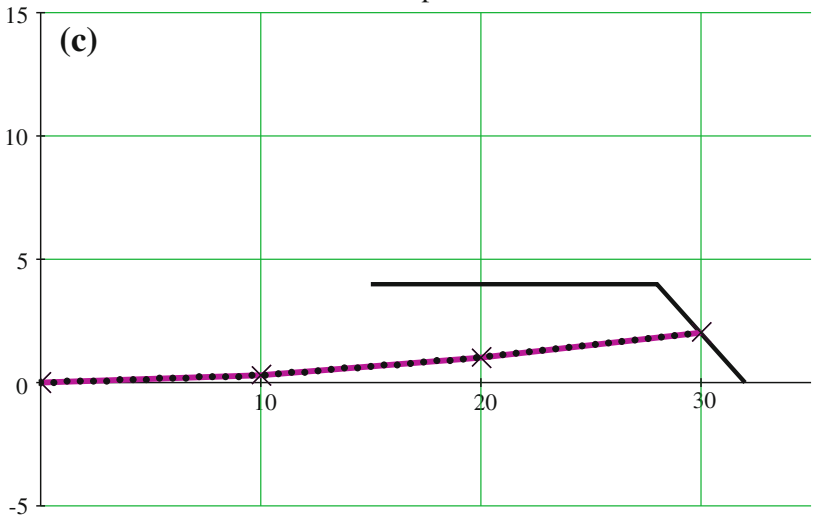

Fig. 3 The beam deflection: a without contact conditions (first iteration), b with contact conditions (second iteration), $\mathbf{c}$ the final beam deflection (third iteration). Dots denotes beam deflection in previous Newton iteration step

The negative result for $\epsilon_{1}$ seems surprising but note that it actually signalizes that the normal contact reaction force at node 3 is expected negative. In other words, it says that the constraint at node 3 is in fact inactive (in order to fulfill constraint it is required to pull the node up to the contact surface), even though it has been detected as active in the previous iteration. Hence, we eliminate this constraint by setting $\epsilon_{1}=0$ in the second iteration. 
Applying this result in the system of Eq. (41) we obtain the following solution for the nodal displacement vector:

$$
\Delta \mathbf{u}=\left[\begin{array}{r}
-0.001 \\
-1.277 \\
-0.230 \\
-0.002 \\
-4.295 \\
-0.368 \\
-0.002 \\
-8.283 \\
-0.414
\end{array}\right], \quad \mathbf{u}=\tilde{\mathbf{u}}+\Delta \mathbf{u}=\left[\begin{array}{r}
-0.001 \\
0.297 \\
0.056 \\
-0.002 \\
1.038 \\
0.089 \\
-0.002 \\
2.002 \\
-0.100
\end{array}\right] .
$$

The approximate beam deflection after the second iteration, Eq. (44), is shown in Fig. 3b. The errors in contact modeling (penetration of the rigid surfaces by contact nodes) are

$\mathbf{D u}-\hat{\mathbf{u}}=\left[\begin{array}{c}- \\ 3.678 \cdot 10^{-8}\end{array}\right]$.

This means that the inaccuracy is much lower than the assumed allowed level $\delta=0.0001 \mathrm{~mm}$.

In the next iteration we repeat the computations for $\tilde{\mathbf{u}}$ set to the current approximate solution (44) and for one active constraint ( $M=1$, matrix $\mathbf{D}$ is reduced to only its second row). To compute the penalty factor $\epsilon_{2}$ we again use (20) and obtain

$\epsilon_{2}=227.8$

Note that this value is only five times higher than the highest diagonal term in $\mathbf{K}$, i.e. it is significantly lower than it would be assumed in the classical penalty method. The new approximate nodal displacement vector $\mathbf{u}=\tilde{\mathbf{u}}+\mathbf{u}^{\prime}$ resulting from (41) is

$\mathbf{u}=\left[\begin{array}{c}0.001 \\ 0.297 \\ 0.056 \\ 0.002 \\ 1.038 \\ 0.089 \\ -0.002 \\ 2.002 \\ 0.100\end{array}\right]^{T}$

The contact constraint is fulfilled with an error equal exactly of $0.0001 \mathrm{~mm}$ which value is assumed as accepted contact modeling inaccuracy. As the norm of residual forces is 0.39 . $10^{-6}$, the vector (47) is considered the final solution of the problem. The final beam deflection is shown in Fig. 3c.

The determinant of the penalized stiffness matrix (in the last Newton step, which determines the solution accuracy) is $\left|\mathbf{K}+\mathbf{D} \boldsymbol{\epsilon} \mathbf{D}^{T}\right|=26.93 \cdot 10^{6}$, i.e. it is considerably higher than for the system without contact constraints, $|\mathbf{K}|=459.7$. Note, however, that the matrix condition number is cond $(\mathbf{K}+$ $\left.\mathbf{D} \boldsymbol{\epsilon} \mathbf{D}^{T}\right)=4.81 \cdot 10^{3}$ which is even less than in that case, cond $(\mathbf{K})=45.72 \cdot 10^{3}$. This decrease of the matrix condition number by one order of magnitude may be considered as lucky coincidence-its values corresponding to different geometries of rigid surfaces may be significantly higher. But even in the worst case the condition number does not exceed the order of magnitude of $10^{4}$ which means that it at least remains at the same level as in the unconstrained system. For comparison, the condition number for the classical penalty approach with $\epsilon_{c}=42 \cdot 10^{3}$ results in the condition number of the penalized matrix cond $\left(\mathbf{K}+\mathbf{D} \boldsymbol{\epsilon}_{c} \mathbf{D}^{T}\right)=841.5 \cdot 10^{3}$ which is significantly higher than in the unconstrained system and in our modified method.

The numerical example has also shown another important advantage of the presented algorithm in comparison with the classical method. Namely, it allowed to detect inactive contact constraint at node 3 after the first iteration [see Eq. (43)], even if it has been activated by the approximate solution computed in this iteration. Negative penalty factor computed for this node indicates that it is attracted to the rigid surface (the normal reaction is negative). In other words, this node does not in fact penetrate the surface and contact constraint should be considered inactive. In the classical method, we would have to assume contact constraint active at the moment and only after the next iteration learn that it should be disactivated. Here, we were able to start the second iteration with the already correct set of active constraints which allowed to save one equilibrium iteration.

\subsection{Deep drawing of a plastic sheet}

The numerical example is a deep drawing of a sheet. The drawing parameters and geometry of tools are taken from the benchmark proposed by Woo in [18]. The geometry of the sheet and tools are presented in Fig. 4.

The sheet was divided into 1800 triangular finite elements with the DKT shell kinematical formulation (6 d.o.f. per node) [17]. The viscoplastic material model with isotropic hardening was assumed and the rigid-plastic formulation (flow approach) was employed. The Coulomb friction model ( $\mu=0.162$ ) was assumed. The sheet thickness is $0.81 \mathrm{~mm}$.

In this paper we will focus only on the contact modeling. The velocities, displacements and sheet thickness are consistent with [18].

There are three cases considered. In the first case the penalty factor is assumed constant, $\epsilon=\epsilon_{c}=10^{6}$. This value allows to obtain the geometric inaccuracy in contact modelling (penetration) at the level $0.01 \mathrm{~mm}$. The two other cases employ the presented modification of the penalty method with the assumed contact inaccuracy levels (for all contact constraints) at 0.01 and $0.001 \mathrm{~mm}$, respectively.

The simulation was divided into 200 equal time steps. In each step a nonlinear problem of equilibrium was solved in Newton iterations. With classical penalty approach the total 

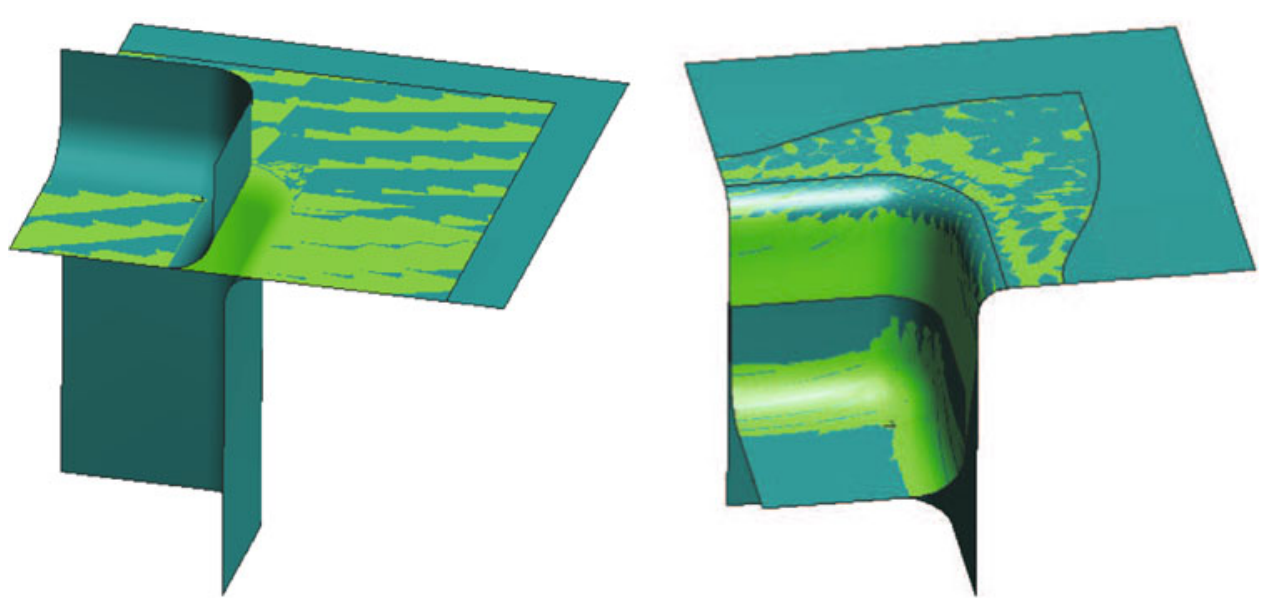

Fig. 4 The benchmark test of a deep drawing: initial geometry (left) and the final shape (right)
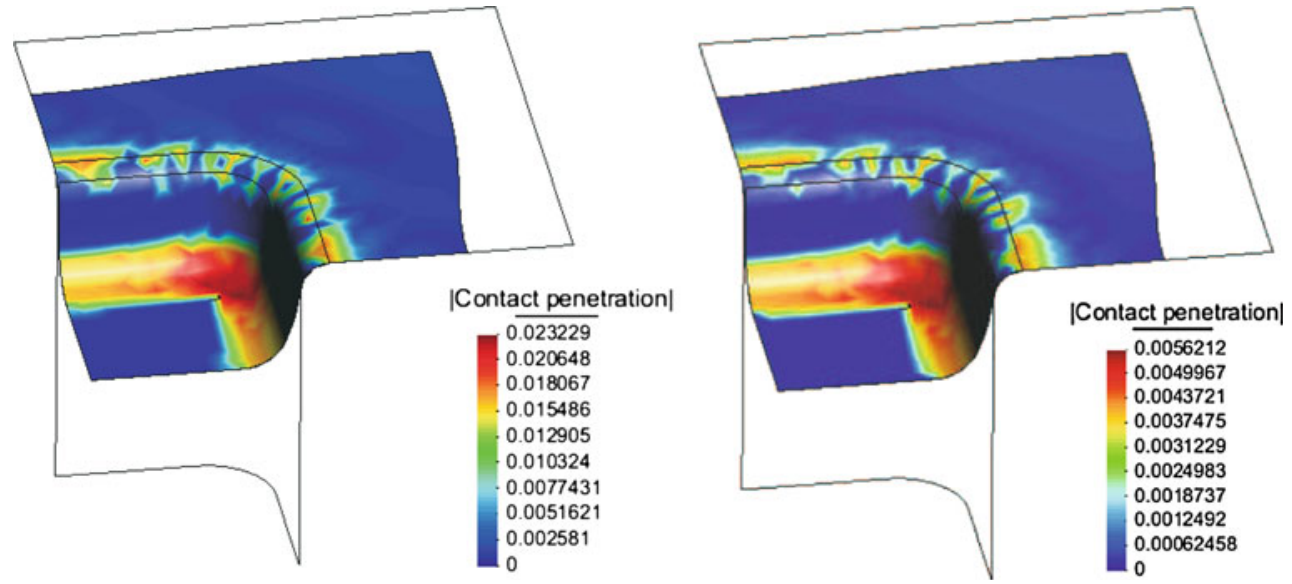

Fig. 5 The drawing benchmark, the contact penetration depth field: with contact modeling accuracy $\delta=0.01($ left $)$ and $\delta=0.001($ right $)$

number of Newton iterations was 963, including 3 steps in which after 25 iterations convergence was not achieved. In cases of presented modification of the penalty approach, the total number of iterations was 836 and 883 , respectively, with no non-converged steps. This means that the computation time does not increase due to the proposed modification.

Figure 5 presents the contact modeling inaccuracy, i.e. the penetration depth of sheet nodes into rigid tools, for the two assumed levels of inaccuracy. In each case the pictures present the results for the step for which the worst inaccuracy was detected. We can see that, although in both cases the penetration values locally exceed the assumed inaccuracy (which is not surprising as the estimation of the penalty factors is based on a number of approximations), its order of magnitude is preserved. For example, the highest penetration depth for $\delta=0.01$ is about 0.023 in this step, but in other steps penetrations are much lower (the average of the maximum penetration values over all steps is 0.002).

The condition number of the main system of equations in the described cases was also examined. Figure 6 presents the chart of the condition number of the penalized tangent matrix in the consecutive Newton iterations. Numbers near lines denote time steps. The chart presents the first 10 time steps. The blue dotted line corresponds to simulation with classical penalty approach (with constant penalty factor) while the green solid line denotes the simulation with the presented modified approach for $\delta=0.01$. We can see that especially in the first time step the condition numbers in the classical approach are significantly higher than in modified penalty algorithm. These ill-conditioning of the main matrix is due to relatively small velocities of blank nodes which results in ill-conditioned viscosity matrix. The modified penalty approach appears to improve this undesired feature. As a result, the number of Newton iterations in this time increment is smaller. In subsequent stable time steps (from the third step up) the condition number of the main system of equations remains still better for the modified approach that in the classical method, although the differences are not that high.

A parametric study was made in order to test the influence of mesh density on the computed penalty factors. Seven structured meshes consisting of 900, 1600, 1800, 2500, 3600, 


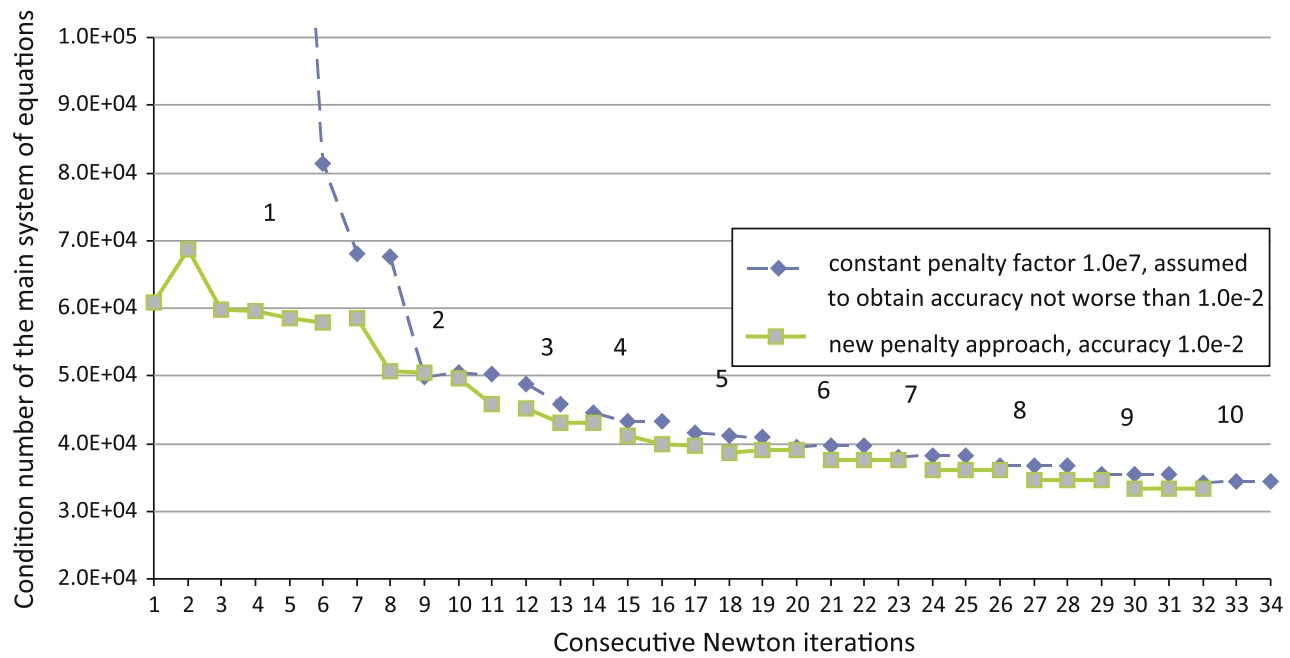

Fig. 6 Matrix condition number during consecutive Newton iteration steps, numbers on chart denotes time steps

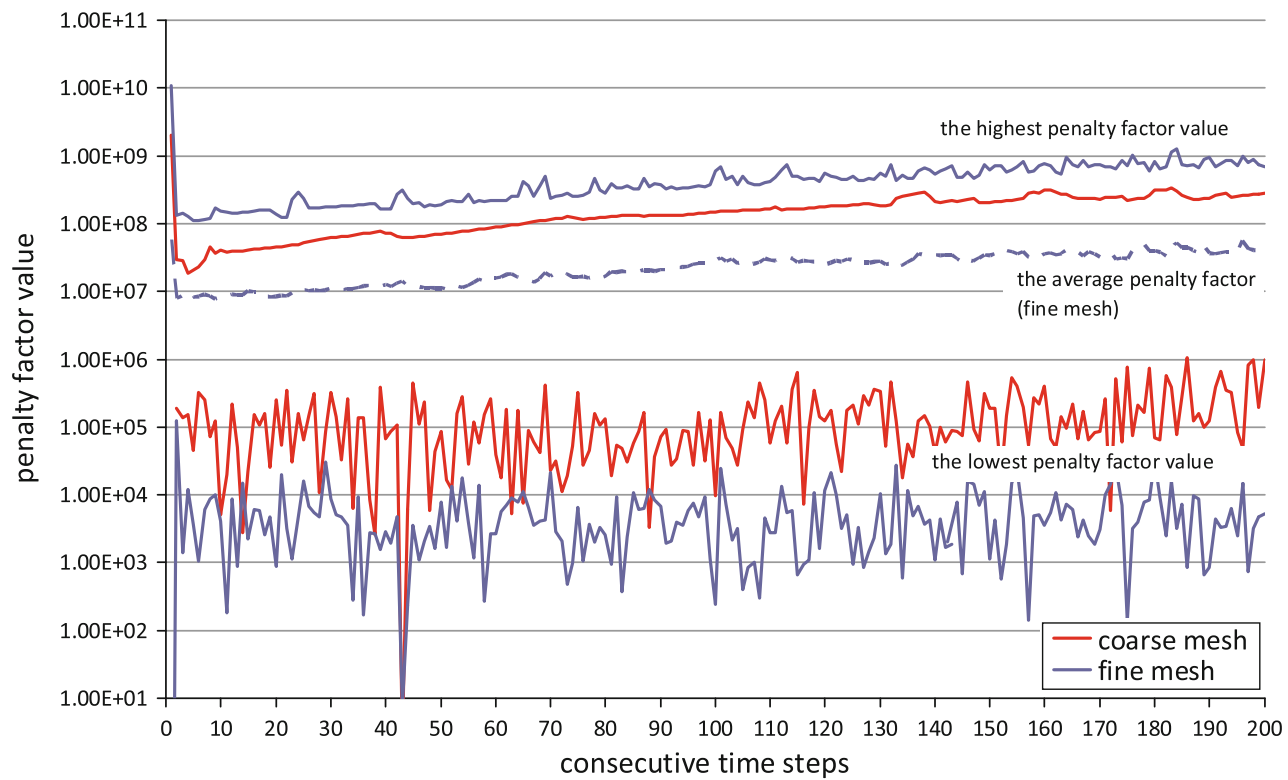

Fig. 7 The highest, lowest and the average penalty factor in consecutive time steps

4900 and 6400 finite elements (triangular with linear shape functions elements) were examined. It should be noticed that the difference of final sheet thickness between models divided to 1,800 elements and 6,400 elements is less than $5 \%$. The highest, lowest and the average penalty factor values in consecutive simulation steps are shown in Fig. 7. The red line denotes coarse (900 elements) mesh, the blue line corresponds to very dense (6,400 elements) mesh. The dashed line refers to the average penalty factor in the case of the dense mesh. We can see that the penalty factors grow with increasing mesh density, but the sensitivity of the computed penalty factors with respect to finite element size is very low. The dif- ference between the highest penalty values computed for the coarse and dense meshes does not exceed one order of magnitude. In the case of intermediate mesh densities (form 1,600 to 4,900 elements) the highest penalty factors in consecutive time steps take intermediate values between the finest and the coarsest mesh cases. The average penalty factor value are nearly the same in all the cases.

\section{Discussion}

It has been shown that the estimated values of penalty factors can be computed from Eq. (20) (or in same cases 
(21-22)). We have also shown that exact values of penalty factors cannot be computed, since the values of displacement corrections $\Delta u_{j}$ in Eq. (20) are not known and a rough approximation has to be used instead.

There are other reasons why the computation of $\epsilon_{k}$ is only approximate. One of them is that $\Delta q_{i}$ in Eq. (20) is in fact unknown, too. This array contains residual forces, i.e. the external load forces diminished by the internal forces that are supposed to equilibrate them. We assumed that all of them should be known at the moment, but note that the friction forces (which are external loads as well) cannot be computed before the contact constraints are considered in the system of equations. Here we have neglected them when building the formulae (20) for penalty factors, although one could also consider here assuming values computed in the previous iteration.

There is one more issue that requires a comment. When differentiating the potential energy functional (4) we have assumed that $\epsilon$ is a constant matrix. Later, from our derivations it appeared that it is evaluated on the basis of the solution (or at least its current approximate). Hence, to be strict, one should consider the generalized form of Eq. (6) in which an additional term containing the derivative $\partial \epsilon_{k} / \partial u_{i}$ appears. This term is, however, neglected in the present formulation which can be justified by the fact that the derivative in this term would have to be multiplied by a squared norm of the vector Du $-\hat{\mathbf{u}}$ which can be considered a small factor.

The above drawbacks of the formulation do not mean that the estimates of penalty factors computed this way cannot be successfully used in real computations. Our numerical examples demonstrate that the method is sufficient to obtain penetrations of the same order of magnitude as the assumed allowed inaccuracy $\delta$. In our tests only in a few time steps and locations the calculated penetrations exceeded $\delta$. In most cases they were significantly (even about $95 \%$ ) smaller then the assumed allowed penetration level.

It must be admitted that, due to the mentioned approximations introduced at the stage of computation of penalty factors, it may happen that the presented method is not likely to lead to a good result, at least in the first equilibrium iteration(s) when the proposed approximation of $\Delta u_{i}$ may appear too rough. In such cases, as it was mentioned earlier, one may choose to use the classical penalty approach and only in the following iterations switch to the modified formulation presented in this paper. On the other hand, if the use of the presented algorithm in a particular computational problem appears to significantly affect the convergence rate in the equilibrium iterations (which may happen due to the mentioned neglection of certain terms in the problem derivation), one may consider switching to the classical constant penalty factor method during the iteration procedure or freezing the once computed penalty factors starting from a certain equilibrium iteration.

\section{Conclusions}

The presented method allows to efficiently adjust values of the contact penalty factors to the actual system of equations and to prescribed tolerance of inaccuracy in fulfillment of contact constraints. The contact modeling is controlled by a directly defined value of maximum allowed penetration (contact modeling inaccuracy) rather than an 'indirect' penalty factor that is indeed difficult to be estimated by a FE code user. This property makes the presented penalty approach comparable to the accuracy of the Lagrange multipliers method, but with much smaller requirements regarding computer resources.

We have also shown that the presented algorithm for adaptive estimation of penalty factors allows to detect the contact constraints that appear to be active but should eventually be deactivated. These constraints are indicated by a negative value of the computed penalty factor. This means that the normal contact reaction required to maintain the node on the contact surface is expected negative (the node is artificially dragged to the contact surface). In such a case, the penalty factor is set to zero which means deactivation of the considered constraint. This feature allows to decrease the number of equilibrium iterations and save the computation time.

The method can improve conditioning of the system matrix compared to classical penalty approach, especially in cases when the base (unconstrained) system matrix is illconditioned itself. This allows to avoid numerical problems in finding accurate solutions of the contact-penalized systems of equations. It should be noticed that computed penalty factors are nearly independent on model discretization.

Acknowledgments This study was partially financed by European Regional Development Fund within the framework of the Innovative Economy Programme, Project Number POIG.01.03.01-14-209/09.

Open Access This article is distributed under the terms of the Creative Commons Attribution License which permits any use, distribution, and reproduction in any medium, provided the original author(s) and the source are credited.

\section{References}

1. Wriggers P (2002) Computational contact mechanics. Chichester, Wiley

2. Oñate E, Zienkiewicz O (1983) A viscous shell formulation for the analysis of thin sheet metal forming. Int J Mech Sci 25:305-335

3. Agelet de Saracibar C (1990) Finite element analysis of sheet metal forming processes. PhD thesis, Universitat Politecnica de Catalunya (in Spanish)

4. Oñate E, de Saracibar CA (1990) Analysis of sheet metal forming problems usig a selective bending-membrane formulations. Int J Num Meth Eng 30

5. Sosnowski W, Bednarek T, Kowalczyk P (2010) Stability and uniqueness of flow approach algorithm in sheet metal forming simulations. Comput Methods Mater Sci 10(1):30-36 
6. Nour-Omid B, Wriggers P (1987) A note on the optimum choice for penalty parameters. Commun Appl Numer Methods 3:581-585

7. Barlam D, Zahavi E (1999) The reliability of solutions in contact problems. Comput Struct 70:35-45

8. Pantano A, Averill RC (2002) A penalty-based finite element interface technology. Comput Struct 80:1725-1748

9. Mik M-S, Choi D-H (2000) A new penalty parameter update rule in the augmented lagrange multiplier method for dynamic response optimization. KSME Int J 14:1122-1130

10. Zavarise G, Wriggers P, Stein E, Schrefler B (1992) Real contact mechanisms and finite element formulation-a coupled thermomechanical approach. Int J Numer Methods Eng 35:767-785

11. Zavarise G, Wriggers P, Stein E, Schrefler B (1992) A numerical model for thermodynamical contact based on microscopic interface laws. Mech Res Commun 19:173-182

12. Paggi M, Barber J (2011) Contact conductance of rough surfaces composed of modified rmd patches. Int J Heat Mass Transf 4:46644672
13. Zavarise G, Lorenzis LD, Taylor RL (2012) A non-consistent startup procedure for contact problems with large load-step. Comp Meth Appl Mech Eng 205(208):91-109

14. Luenberger D (1984) Linear and nonlinear programming, 2nd edn. Addison Wesley

15. Perzyna P (1966) Fundamental problems in viscoplasticity. Adv Appl Mech 9:243-377

16. Oñate E, Agelet de Saracibar C (1992) Numerical modelling of sheet metal forming problems. In: Hartley P, Pillinger I, Sturgess C (eds) Numerical modelling of material deformation processes: research, development and applications. Springer, New York

17. Batoz J, Bathe K, Wo L (1980) A study of three node triangular plate bending element. Int J Num Methods Eng 15:1771-1812

18. Woo D (1968) On the complete solution of the deep drawing problem. Int J Mech Sci 10:83-94 